\title{
Identification and Characterisation of A Locally Isolated Lipolytic Microfungus - Geotrichum candidum
}

\author{
Loo, J.L. ${ }^{1}$ Lai, O.M. ${ }^{2}$, Long, K. $^{3}$ and Ghazali, H.M. ${ }^{{ }^{*}}$ \\ ${ }^{1}$ Department of Food Science, Faculty of Food Science and Technology, \\ University Putra Malaysia, 43400 Serdang, Selangor, Malaysia. \\ ${ }^{2}$ Department of Bioprocess Technology, Faculty of Biotechnology and Biomolecular Sciences, \\ University Putra Malaysia, 43400 Serdang, Selangor, Malaysia. \\ ${ }^{3}$ Biotechnology Division, Malaysia Agricultural Research and Development Institute, \\ P.O. Box 12301, 50774 Kuala Lumpur, Malaysia. \\ Email: hasanah@putra.upm.edu.my
}

\begin{abstract}
A strain of Geotrichum candidum was successfully isolated from local soil samples and some of its characteristics determined. The strain produces lipase that hydrolysed palm olein on a selective agar incorporated with Nile Blue sulphate and palm olein. Suspension culture was used to study the growth and lipolytic activity. The results showed that the highest dry cell mass of G. candidum was achieved after 96 hours of growth when $2 \%$ (v/v) seed culture was used as inoculum. Using spore suspension as an inoculum was not favourable since less yield of mycelia mass was obtained at peak of log phase and the culture exhibited relatively low lipolytic activity and efficiency of hydrolysis. The maximum amount of free fatty acids $(90.5 \%)$ was released after 48 hours of incubation. However, the highest lipolytic activity, 7.02 mmole FFA/g dry cell was detected at 54 hours. The production of cell-bound lipase of $G$. candidum was concomitant with growth and declined when growth ceased. During the course of growth, changes to palm olein added into the culture medium caused the growth culture to turn into a creamy colloidal emulsion. Towards the end of the incubation period, solid fatty particles were formed. Thin layer chromatographic analysis showed that triacylglycerols in the palm olein were rapidly hydrolysed to fatty acids in 18 hours, with no pronounced change in the amounts of 1,2-diacylglycerols and 1,3-diacylglycerols.
\end{abstract}

Keywords: G. candidum, morphology, growth, lipolytic activity

\section{INTRODUCTION}

For the past decade, the oleochemical industry has become one of the rapid growth areas in the economy of Malaysia in terms of magnitude of the production, the diversity and the uses of fatty acids-related products. Malaysia has become a major producer of the basic oleochemicals using the large supplies of indigenous raw material such as palm oil, palm kernel oil and coconut oil (Gunstone, 2004). Refined, bleached and deodorized (RBD) palm olein is easily available in Malaysia, and by applying biotechnological means the functionality of RBD palm olein can be improved to produce more value-added products for the oleochemical industry. Enzymatic hydrolysis of fats using lipases offers an alternative method for fatty acid production due to its mild reaction conditions, as opposed to the conventional Colgate-Emery process that involves high pressure and high temperature splitting (Pantzaris, 1995). By using enzymes that are substrate specific or stereospecific, the hydrolysis process can be directed to yield desirable products that are free of other by-products or contaminants.

Since palmitic and oleic acids are abundant in palm oil, a lipase that discriminates against saturated fatty acids could be of commercial use for the splitting and restructuring of natural triglycerides. The preferential

\footnotetext{
${ }^{*}$ Corresponding author
}

hydrolysis for cis-9 unsaturated fatty acids notably oleic, linoleic, linolenic and palmitoleic acids, has been viewed as industrially useful (Iwai et al., 1973; Shridhar et al., 1991; Shimada et al., 1995). Oleic and linoleic acids are used extensively in food and non-food industry, such as in soap and detergent, surfactant, textile, cosmetic and pharmaceutical industry (Sonntag, 1991). The lipase from $G$. candidum is of interest because of its unique specificity for hydrolysis of cis-9 unsaturated fatty acids in relative to both palmitic acid and stearic acid (Alford et al., 1964; Charton and Macrae, 1992; Sonnet et al., 1993; Shimada et al, 1995). Strains of the microfungus $G$. candidum have been isolated for lipase production and their substrate specificity has been widely studied (Alford et al., 1964; Tsujisaka et al., 1973; Baillargeon, 1990; Baillargeon and McCarthy, 1991; Sidebottom et al., 1991). In view of $G$. candidum lipase's unique specificity for unsaturated substrates, an attempt was made to isolate and culture the microfungus from local sources. This paper reports on some of the properties and potential development of using the lipase isolated from $G$. candidum. 


\section{MATERIALS AND METHODS}

\section{Materials}

Microbiological media were purchased from Difco, USA and Oxoid, England. Chemicals and organic solvents used were of analytical grade. Palm olein was obtained from a local hypermarket in Sri Serdang, Selangor, Malaysia. Soil samples were collected from an oil palm estate near UPM and were used as sources of lipolytic fungi.

\section{Isolation of lipolytic fungus}

Twenty grams of soil were homogenised in $100 \mathrm{ml}$ sterile distilled water and then filtered through a Whatman No. 4 filter paper. Five milliliters of the filtrate were transferred into a $250 \mathrm{ml}$ Erlenmeyer flask containing $100 \mathrm{ml}$ broth that has been autoclaved at $121^{\circ} \mathrm{C}$ (Hirayama, Hiclave, HVE-50, Japan) for 15 minutes. The composition of the broth was as follows: $1 \%$ glucose, $0.5 \%$ malt extract, $0.5 \%$ peptone, $0.3 \%$ yeast extract and $200 \mathrm{ppm}$ of chloramphenicol. The $\mathrm{pH}$ of the broth was adjusted to 3.7 using $2 \mathrm{~N} \mathrm{HCl}$. Chloramphenicol was added to inhibit growth of undesirable bacteria. Incubation was carried out at $30^{\circ} \mathrm{C}$ and $120 \mathrm{rpm}$. After 48 hours, the culture was serially diluted in $1 \%$ peptone solution before spreading $0.1 \mathrm{ml}$ of appropriately diluted culture broth onto yeastmalt extract agar for growth at $30^{\circ} \mathrm{C}$. Visual examination revealed that $G$. candidum on yeast-malt extract agar was white, velvety (fuzzy) colony that is flat and spreading. However, the colony would turn slimy, with sheen and was semi-transparent after about a week. Colonies that resembled $G$. candidum morphologically were further isolated and purified using streak plate technique until a single, pure colony was obtained.

\section{Strain conservation and preparation of inoculum}

Single isolated colonies of $G$. candidum strain were maintained on YMA (yeast-malt extract agar) slant incubated at $30^{\circ} \mathrm{C}$ for 72 hours, then stored at $4^{\circ} \mathrm{C}$. Subculture was carried out every month to ensure continuity of pure culture and to maintain cell viability. For seed culture preparation, a loopful of the microorganism grown on YMA slant was transferred into a universal bottle containing $20 \mathrm{ml}$ yeast extract $(0.5 \%)$, peptone $(1 \%)$ and glucose $(2 \%)$ broth at $\mathrm{pH} 7.2$ and incubated at $30^{\circ} \mathrm{C}$ for 24 hours. Arthrospores were produced in a similar method but with longer incubation time $\left(7\right.$ days) at $30^{\circ} \mathrm{C}$. The liquid medium was then filtered through Whatman No. 4 filter paper aseptically to remove the mycelium. The filtrate contained the spores. Spore density was determined by haemocytometer. After serial dilution in $0.1 \%$ peptone solution, the spore suspension contained about $10^{6}$ spores per $\mathrm{ml}$.

\section{Screening for lipolytic activity}

An oil agar medium similar to that described by El-Azzabi et al. (1981) was used to detect lipolytic activity. The composition of the El-Azzabi et al. (1981) medium was 8 $\mathrm{ml}$ palm olein, $4 \mathrm{ml} 0.1 \%$ aqueous Nile Blue sulphate, 0.5 $\mathrm{g}$ tryptone, $2.0 \mathrm{~g}$ agar and deionised water to make the volume up to $100 \mathrm{ml}$. Ghazali (1990) reported that freshly prepared oil agar containing palm olein had grayish pinkish-blue color while aged agar had an orange-pink color. The microorganisms were point inoculated onto the palm olein-Nile Blue sulphate agar medium and incubated at $30^{\circ} \mathrm{C}$. Observations were carried out daily for growth and changes that would indicate lipolysis. Lipolytic activity was assessed by the formation of insoluble blue droplets entrapped within the agar underneath the yeast colony caused by the release of free fatty acids (FFA) into the initially orange-pink color agar.

\section{Identification of isolates}

Positive colonies from the oil agar medium were further studied for their physiological and morphological growth, and compared to previous reports (Carmichael, 1957; Frazier and Westhoff, 1978; Domsch et al., 1980; Ghazali, 1990). Techniques and methods for studying the various features were as described by van der Walt and Yarrow (1984). The scheme for the identification of food borne yeast reported by Deak and Beuchat (1987) and taxanomic study of yeast by Kreger-van Rij (1984) and Lodder (1971) were used in the identification of the isolates.

\section{Determination of growth}

The cultivation of $G$. candidum was performed in a nutrient medium comprising $0.1 \%$ peptone, $0.2 \%$ glucose, $2 \%$ yeast-extract, $0.1 \%$ dipotassium hydrogen phosphate and $0.5 \%$ ammonium sulphate. The $\mathrm{pH}$ of the medium was adjusted to 7.2 with $2 \mathrm{~N} \mathrm{NaOH}$ before autoclaving at $121^{\circ} \mathrm{C}$ for 15 minutes. Palm olein used for inducing lipase production was pre-sterilised by dry heating at $100^{\circ} \mathrm{C}$ for 1 hour and then added aseptically to the liquid medium at $2 \%(\mathrm{w} / \mathrm{v})$ concentration at the start of the experiment. After inoculation with 24-hour seed culture or 7-day spores and addition of the sterilized oil, the culture flask was incubated at $30^{\circ} \mathrm{C}$ with shaking at $120 \mathrm{rpm}$. Samples $(20$ $\mathrm{ml}$ ) were withdrawn at regular time intervals to determine the $\mathrm{pH}$ of growth medium, cell growth and lipase activity. Another flask containing the above growth medium and oil, without addition of inoculum was run concurrently with the test to check for non-enzymatic hydrolysis of the added oil.

The growth culture was filtered through a preweighed Whatman No. 4 filter paper to collect the mycelia. The resulting filtrate (spent culture medium) was centrifuged at $8000 \mathrm{rpm}$ at $4^{\circ} \mathrm{C}$ for 15 minutes in a high speed centrifuge (Beckman, J2-21M/E Centrifuge, USA) to collect residual mycelium. The total mycelium was washed several times with distilled water and hexane to 
remove the fat. Growth was determined gravimetrically by placing the fungal mycelium in an oven at $70^{\circ} \mathrm{C}$ until a constant weight was obtained. Growth was expressed as grams of the dried mass per liter of growth culture.

\section{Extraction of residual lipid}

The filtrate or spent culture medium was transferred into a $250 \mathrm{ml}$ separatory funnel and the lipid material extracted with $150 \mathrm{ml}$ diethyl ether:ethanol (2:1). The organic phase was washed with distilled water and then filtered through Whatman No. 4 filter paper containing anhydrous sodium sulphate. The solvent was removed in vacuo using rotary evaporator (Tokyo Rikakikai Co. Ltd. and Eyela SB-35) and lipid residue was dried in $60^{\circ} \mathrm{C}$ oven for 3 hours to remove residual hexane.

\section{Determination of free fatty acids and lipase activity}

The FFA content was determined according to PORIM Test Method (Siew et al., 1995) by direct titration with 0.05 $\mathrm{N} \mathrm{NaOH}$ to a phenolphthalein end equivalent point (EQP). The oil sample was first dissolved in $50 \mathrm{ml}$ of neutralized iso-propanol. The acid value was expressed as percent of FFA liberated from the total fatty acid available in a given amount of oil. Lipolytic activity of $G$. candidum during growth was quantified by micromole of FFA in the residual lipids extracted per gram of dry mycelium.

\section{Detection of products of lipolysis}

The separation of FFA, mono- ,di- and triacylglycerols in the lipid material obtained following extraction was performed by thin-layer chromatography (TLC) on silica gel G60 (Merck, UK) plates impregnated with $1.2 \%$ boric acid in absolute ethanol $(1: 1, v / v)$ as the stationary phase. An aliquot of $3 \mu \mathrm{l}$ of molten lipid extract was spotted at 2 $\mathrm{cm}$ from the lower side of the plate in $10 \mathrm{~mm}$ length band. The mobile phase of the system comprised nhexane:diethyl ether:acetic acid in 70:30:1 ratio. The plate was then placed in a glass chamber filled with iodine vapour to develop the chromatogram where lipid components were reveals as yellow spots on the white background. Highly purified samples of FFA (oleic acid), monoacylglycerol (1-mono-stearoyl-rac-glycerol), diacylglycerols (1,2- and 1,3-diolein) and triacylglycerol (triolein) were also spotted onto the plate to locate the relative positions of the hydrolysed products.

\section{RESULTS AND DISCUSSION}

\section{Characteristics of G. candidum}

This paper deals mainly with the screening for $G$. candidum from local soil and subsequently cultivating its growth to produce cell-bound lipase. During screening, ability of a microbial isolate to hydrolyse oil (palm olein) was shown by the formation of insoluble blue droplets entrapped underneath microbial colony. This was clearly visible when the colonies were viewed from the reverse side of the agar plates. Colonies that were negative for lipase production did not show such formation. Among the 70 well-isolated colonies obtained and screened for lipolytic activity, only 15 were found to be lipolytic. Positive colonies that resembled $G$. candidum morphologically and microscopically were further isolated and purified, until a pure, single colony was obtained. The $G$. candidum strain was identified with reference to the simplified key devised by Deak and Beauchat (1987) and taxanomic study of yeast by Kreger van-Rij (1984) and Lodder (1971). Table 1 shows some of the physiological and biochemical characteristics of the G. candidum strain. It was found that the strain fermented sugars such as glucose and galactose, and was resistant to cycloheximide. However, nitrate, urea and gelation were not hydrolysed by this microfungus. $G$. candidum has been isolated from many different substrates in the past, including milk, bulbs, fruits, human and animal material, and industrial sources. An account of the genus is provided by de Hoog et al. (1986). Confirmation of the strain was done by CABI Bioscience Center, Egham, Surrey, United Kingdom.

Table 1: Physiological and biochemical characteristics of the $G$. candidum strain

\begin{tabular}{cc}
\hline Characteristics & Observations \\
\hline Fermentation of sugars & \\
Glucose & + \\
Galactose & + \\
Resistence to Cycloheximide & \\
100 ppm & $r$ \\
1000 ppm & - \\
Production of acetic acid & - \\
Nitrate utilization & - \\
Splitting of urea & + \\
Growth at $30^{\circ} \mathrm{C}$ & - \\
Growth at $37^{\circ} \mathrm{C}$ & - \\
Growth on acetic acid agar & - \\
Liquefaction of gelatin &
\end{tabular}

Figures 1 and 2 show a single, purified colony after growing for 24 hours. It was white, soft, velvety and with a central umbo that appeared to radiate outward; some tiny and fine mycelium were also observed. With prolonged growth at $30^{\circ} \mathrm{C}$, the colony turned wet and semitransparent. When grown in nutrient broth, G. candidum grew into pellicular type structure that floated on the surface of the broth (Figures $3 \mathrm{~A}$ and $3 \mathrm{~B}$ ). It would sediment as the culture aged. After approximately 4 days, arthrospores were formed by fragmentation of the long mature hyphae at the septa and terminal cells (Figure 3C) as a mode of vegetative cells reproduction. These morphological features were similar to those observed by Charton and Macrae (1992) in G. candidum CMICC 335426 , but the growth cycle from germination to sporulation was much shorter, which was 20 hours as compared to the strain understudied (4 days). 


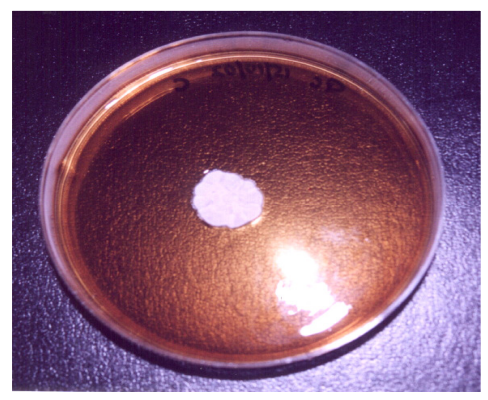

Figure 1: Morphological growth of $G$. candidum on yeast malt extract agar after 24 hours.

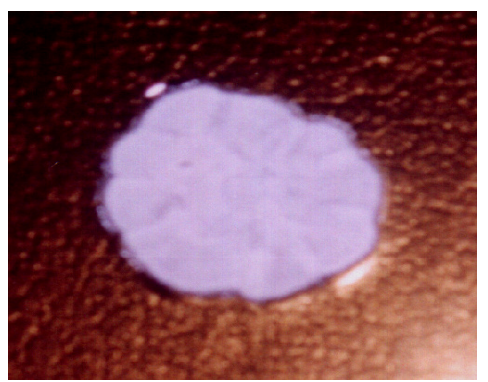

Figure 2: Characteristics of G. candidum colony: white, velvet, flat and spreading, often with a central umbo.
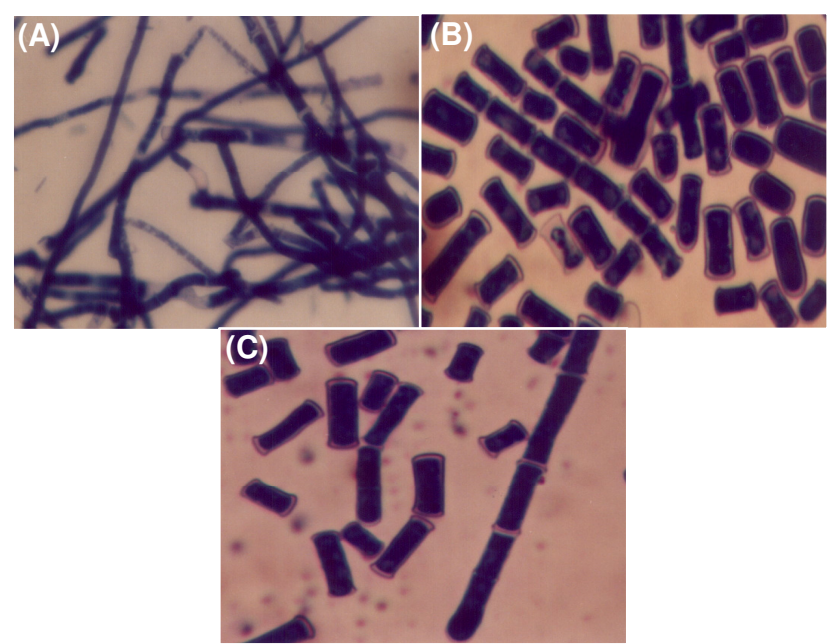

Figure 3: Morphological growth of $G$. candidum in liquid broth culture.

(A): Growth of $G$. candidum in YM broth consist of hyphae and arthrospores (x 100 magnification).

(B): Arthrospores surrounded by thick cell wall ( $x 400$ magnification).

(C): Fragmentation of hyphae to form arthrospores (x 400 magnification).

\section{Effect of inoculation on growth}

Cell growth was studied using both 7-day spores and 24hour seed culture as inocula. Three inoculum sizes were used: $2 \%, 5 \%$ and $10 \%(\mathrm{v} / \mathrm{v})$ from a spore suspension of 1 $\times 10^{6}$ arthrospores per ml. Figure 4 shows that maximum growth in terms of dry cell mass was obtained with $2 \%$ (v/v) spore after 72 hours (6.3 g/L dry weight). In this study, it was observed that the mycelia mass of $G$. candidum did not increase significantly with an increase in inoculum size of spores. The time course in Figure 4 shows that although $2 \%$ and $5 \%$ spore size shared a similar rate of growth for the first 24 hours, the latter did not produce more biomass even though both achieved the highest growth after 72 hours. Increasing the spore inoculum size to $10 \%$ also did not result in higher biomass. In fact a longer lag phase (24 hours) was observed before maximum growth was achieved, probably because a longer dormant period was required to transform most of the arthrospores into the vegetative cells when the spores density increased. Therefore, the inoculum size of $2 \%(\mathrm{v} / \mathrm{v})$ was chosen for the subsequent experiments. In a report by Jacobsen et al. (1989), maximum dry weight $(5.9 \mathrm{~g} / \mathrm{L})$ was reached in much shorter time, which was after 22 hours of growth when 20 $\mathrm{ml}$ of spore suspension (about $10^{7}$ spores $\mathrm{ml}^{-1}$ ) was inoculated in $800 \mathrm{ml}$ culture medium. The variations in results could be due to strain differences.

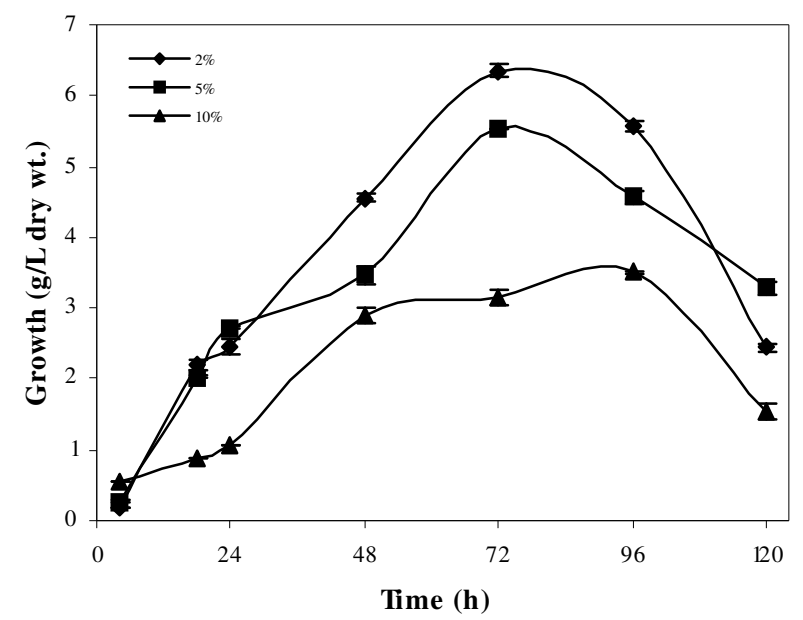

Figure 4: Growth of $G$. candidum in liquid culture medium inoculated with different spore inoculum size.

In order to improve the biomass production, cell growth was further investigated using 24 hour old-seed culture to inoculate the growth medium. The growth of $G$. candidum varied when using $2 \%(\mathrm{v} / \mathrm{v})$ spore suspension and $2 \%(\mathrm{v} / \mathrm{v})$ seed culture as inoculum as shown in Figure 5. Even though the former achieved a higher mycelium yield in the first 3 days, growth ceased gradually after that period. On the other hand, a higher dry cell mass of $12.2 \mathrm{~g} / \mathrm{L}$ was achieved at 96 hours after a longer stationary 
phase for medium inoculated with $2 \%(\mathrm{v} / \mathrm{v})$ seed cultures. This was almost twice the maximum amount obtained by spore inoculation. Therefore, for subsequent studies, cultivation of $G$. candidum was performed using only 24hour old seed culture as inoculum to obtain higher mycelium yield. Besides seed culture and spores, fungal mycelia obtained from a 3-day old culture on Sabouraud$4 \%$ malt agar has also reported to be used as a mode of inoculation to cultivate Mucor hiemalis F. hiemalis by Hiol and his co-workers (Hiol et al., 1999).

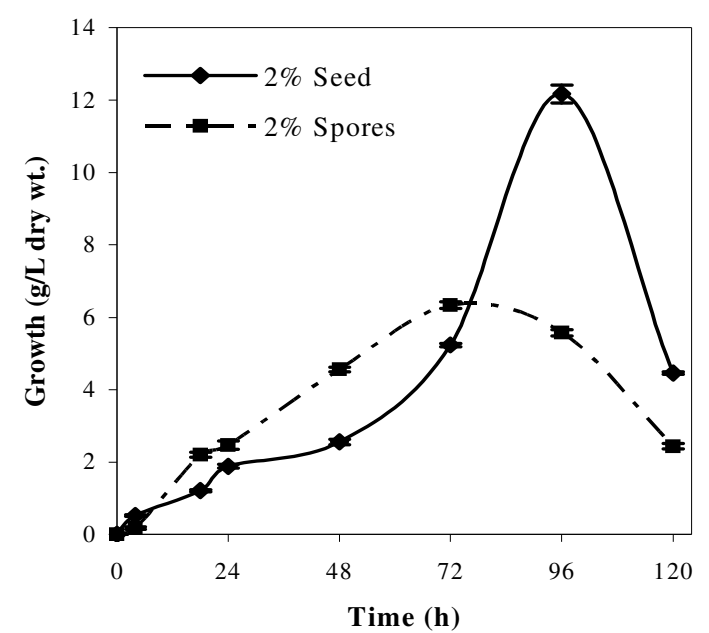

Figure 5: Growth of G. candidum in liquid culture medium inoculated with 24-h seed culture and spore suspension.

\section{Lipolytic activity}

The extracellular lipase of the filamentous fungus $G$. candidum has been described as one of the few known fatty acid-specific lipases preferentially hydrolyse esters of fatty acids with cis-9 double bonds (Alford et al., 1964). The FFA in seed culture-inoculated medium increased in a linear fashion until reaching a plateau at 3.3 milimole or $90.5 \%$ at 48 hours then decreased gradually thereafter as shown in Figure 6. This could mean that the thermodynamic equilibrium of the lipolysis process appeared to be reached after 48 hours. The accumulation of FFA in the growth medium could be an inhibitory factor to the thermodynamic equilibrium of the lipolysis process. Nevertheless, the mycelial cell mass still exhibited positive growth during this period, which suggested that the release of FFA into the growth medium did not limit the growth of the microorganism as demonstrated in Figures 5 and 6.

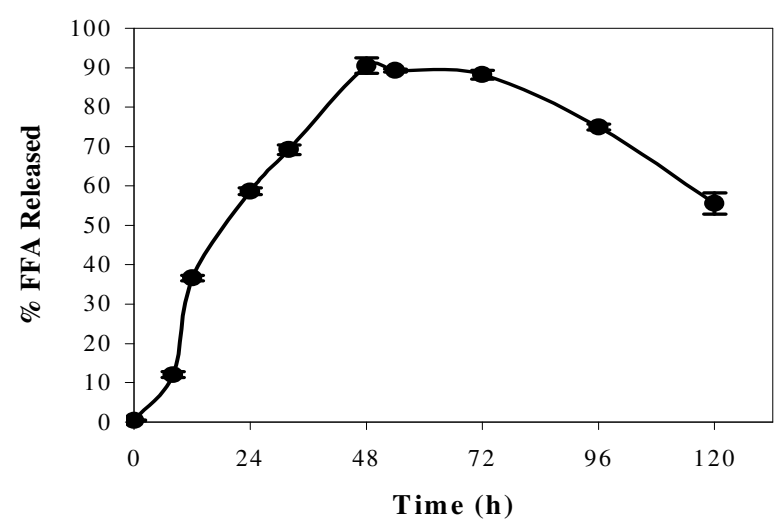

Figure 6: Release of free fatty acids by lipase of $G$. candidum from RBD palm olein in shake-culture broth.

The change in the $\mathrm{pH}$ of the growth medium with time is shown in Figure 7. The $\mathrm{pH}$ decreased sharply from 7.1 to 4.2 for first 24 hours, then increased slightly to 4.9 in the next 24 hours and decreased gradually from there on. The change in $\mathrm{pH}$ during growth of cultures could not be ascribed to release of FFA as the concentration of FFA in the residual lipid extracted from the growth medium was greatest in early stages of culture growth while $\mathrm{pH}$ decline occurred throughout the growth period. The $\mathrm{pH}$ decline could therefore be due to metabolism of the carbon sources, such as yeast extract, glucose and palm olein as explained by Tan and Gill (1984). The results in Figure 8 show that lipid materials in the culture broth remained about $38.7 \%$ after the cultivation period, which indicates that $61.3 \%$ of palm olein was possibly utilized for growth. Cells grew exponentially (Figure 5) in palm oleincontaining medium until most triacylglycerol was exhausted (Figure 11). Much of the lipid material extracted was in the form of FFA especially after about 18 hours of incubations.

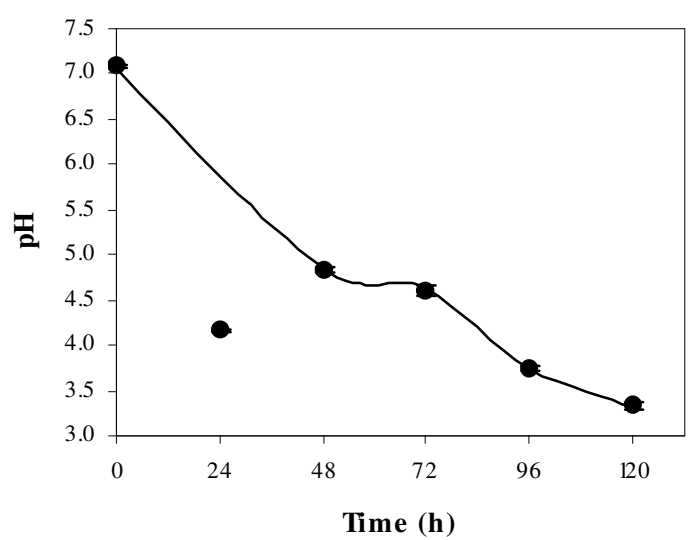

Figure 7: Changes in $\mathrm{pH}$ of liquid medium during growth of G. candidum using $2 \% 24$-hour seed culture. 


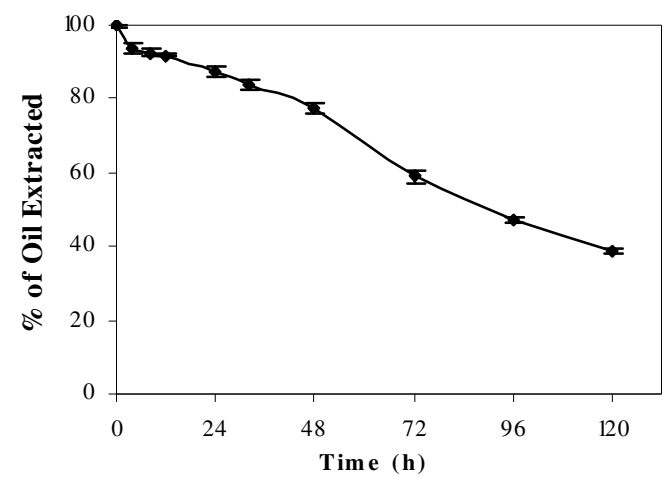

Figure 8: Percentage of residual lipids extracted from liquid medium during growth of $G$. candidum.

In the past, it was found that $G$. candidum required a special component such as lipid in the medium for its lipase production (Alford and Smith, 1965; Iwai et al., 1973 and Tsujisaka et al., 1973). Other microorganisms that produce lipases inducibly are $M$. hiemalis $f$. hiemalis (Hiol et al., 1999) and Candida lipolytica (Sugiura and Isobe, 1975). The lipolytic activity of $G$. candidum was expressed as the amount of FFA released in micromole over the mass of dry mycelium. Figure 9 shows that the highest lipolytic activity, 7.02 milimole/g achieved after 54 hours of growth, was very close to the time when maximum FFA content was produced, which was at 48 hours. The lipase activity then declined steadily. The decrease in media $\mathrm{pH}$ during growth (Figure 7) could also contribute to the loss of activity.

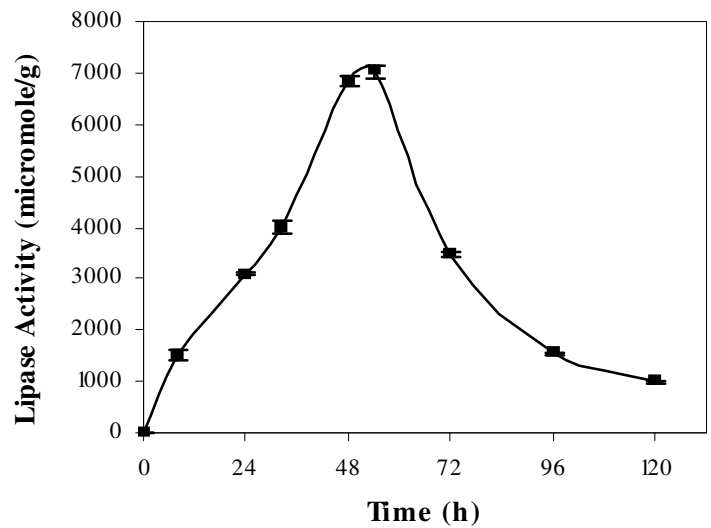

Figure 9: Lipolytic activity of $G$. candidum detected during growth.

The lipase activity was expressed as micromole FFA per gram dry weight.

\section{Detection of hydrolyzed products by thin layer chromatography}

During the course of growth a significant change in the physical appearance of the palm olein added as substrate was observed. The viscosity of the liquid oil in shake- culture broth increased and agglomerated as pellet on the surface of the broth as incubation proceeded. Figure 10 shows the physical appearance of residual lipids extracted from the growth medium, the clear golden liquid turned to creamy colloidal emulsion, then to solid fatty materials towards the end of the incubation period. This is a clear evidence that biotransformation of palm olein had occurred. Solid formation was due to the hydrolysis of triacylglycerol to FFA, especially stearic and palmitic acids, which are solid at $30^{\circ} \mathrm{C}$.

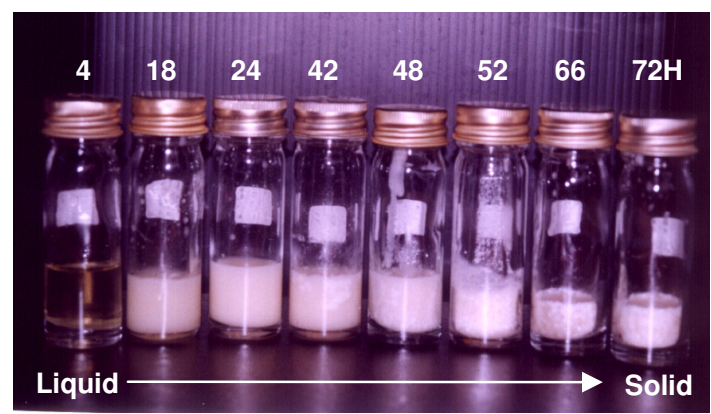

Figure 10: Physical appearance of residual lipids (palm olein) extracted from growth medium.

Thin layer chromatography (TLC) was utilized to follow the progress of lipolysis and to determine the composition of the hydrolysate. The sequence of separation starting from the original spot was: monoacylglycerol, 1,2-diacylglycerol, 1,3-diacylglycerol, FFA and triacylglycerol. Figure 11 shows the concentration changes for substrate and hydrolysis products formed during cultivation of $G$. candidum using $2 \%(v / v)$ of 24 hour-seed culture. Concomitant decline in triacylglycerol and rapid accumulation of FFA, diacylglycerols and monoacylglycerols occurred throughout growth. Triacylglycerols in the palm olein was rapidly hydrolysed to fatty acids at 18 hours, with no prominent change in the amounts of 1,2-diacylglycerols and 1,3-diacylglycerols, based on the relative spot areas on TLC plate (Figure 11). This was because diacylglycerols served only as a transient intermediate in the hydrolytic process.

\section{Efficiency of hydrolysis}

The efficiency of hydrolysis shown in Figure 12 was expressed as the rate of hydrolysis over the rate of growth, where the rate of hydrolysis was the percentage of FFA liberated per hour and the rate of growth was derived from the dry cell mass per hour. From the efficiency of hydrolysis, the ability of the mycelium-bound lipase to release fatty acids from palm olein on a per gram dry weight basis was defined. It was obvious that the culture inoculated with $2 \%$ seed culture achieved far higher efficiency than its spore counterpart. The former attained highest efficiency of hydrolysis after 24 hours, which was equivalent to a FFA production rate of $23.8 \%$ 
per gram dry mycelium. Although there was a positive increase in mycelia cell mass (Figure 5) and FFA (Figure 6 ) up to 96 and 48 hours respectively, the efficiency of hydrolysis of palm olein by the microorganism decreased gradually at 24 hours before picking up again after 96 hours. The increase after 96 hours was probably attributed to transformation of vegetative cells in the culture that involved fragmentation of hyphae into arthrospores.

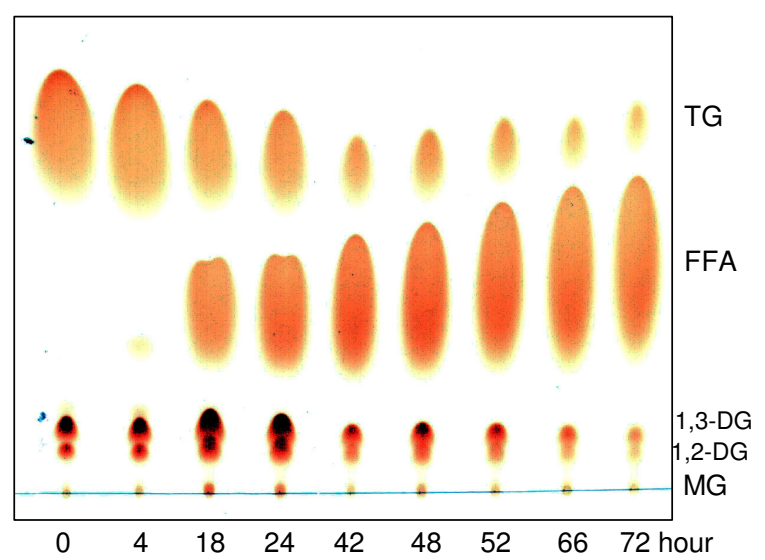

Figure 11: Separation of lipid components in residual lipid extracted from growth medium by thin layer chromatography.

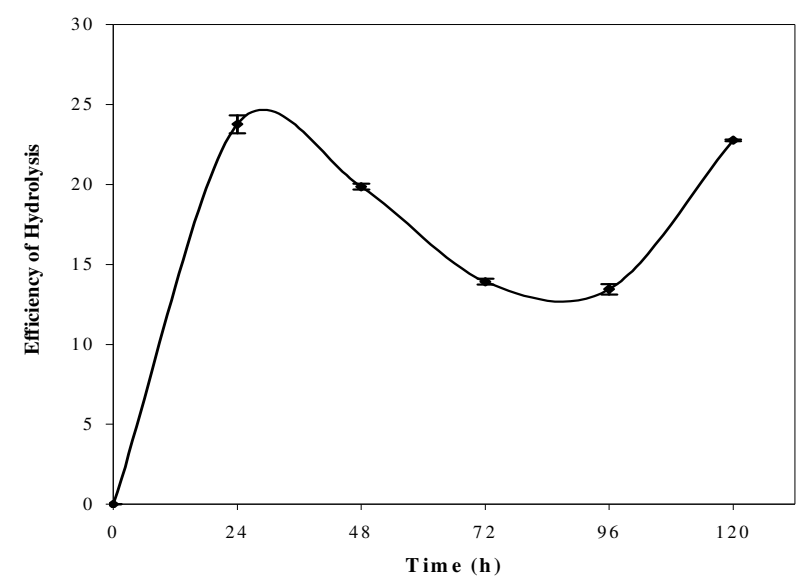

Figure 12: Efficiency of hydrolysis of palm olein by lipase of $G$. candidum during growth. The efficiency was derived from rate of hydrolysis over rate of growth.

\section{CONCLUSION}

G. candidum strain was successfully isolated from local soil and some of its properties and characteristics were determined in our laboratory. The strain produces lipases that hydrolyze palm olein in the selective agar and liquid medium. The results showed that the highest dry cell mass of $G$. candidum was achieved after 96 hours of growth when $2 \%(\mathrm{v} / \mathrm{v})$ seed culture was used as inoculum. Using spore suspension as an inoculation method was not favorable since fewer yields of mycelia mass was obtained at peak of log phase and the culture exhibited relatively low lipolytic activity and efficiency of hydrolysis. Increase in mycelium mass was parallel with depletion in triacylglycerols and accumulation of FFA in the medium. The maximum amount of FFA was released at 48 hours, however highest lipolytic activity was detected at 54 hours. The results proved that the production of cellbound lipase of $G$. candidum was concomitant with growth and declined when growth ceased. Further studies on the properties and potential applications of $G$. candidum lipase are being carried out.

\section{ACKNOWLEDGEMENT}

Financial support by IRPA grant from the government of Malaysia awarded to H.M. Ghazali is gratefully acknowledged and appreciated.

\section{REFERENCES}

Alford, J.A. and Smith, J.L. (1965). Production of microbial lipases for the study of triglyceride structure. J. Am. Oil Chem. Soc. 42(12):1038-1040.

Alford, J.A., Pierce, D.A. and Suggs, P.G. (1964). Activity of microbial lipases on natural fats and synthetic triglycerides. Journal of Lipid Research. 5:390-394.

Baillargeon, B.W. and McCarthy, S.G. (1991). Geotrichum candidum NRRL Y-553 lipase: purification, characterization and fatty acid specificity. Lipids 26(10):831-836.

Baillargeon, M.W. (1990). Purification and specificity of lipases from Geotrichum candidum. Lipids 25(12):841-848.

Carmichael, J.W. (1957).Geotrichum candidum. Journal of Mycologia 49:820-830.

Charton, E. and Macrae, A.R. (1992). Substrate specificities of lipase A and B from Geotrichum candidum CMICC 335426. Biochimica et Biophysica Acta 1123:59-64.

De Hoog, G.S, Smith, M.T. and Gueho, E. (1986). A revision of the genus Geotrichum and its telemorphs. Studies in Mycologia. 29:1-131.

Deak, T. and Beuchat, L.R. (1987). Identification of foodborne yeast. Journal of Food Protection, 50(3):243-264.

Domsch, K.H., Gams, W. and Anderson, T-H. (1980). Compendium of soil fungi. Vol. 1. Academic Press, U.K. p. 348-351.

El-Azzabi, Y.S., Clarke, J.H. and Hill, S.T. (1981). Lipolytic activity of fungi on rapeseed oil. Journal of the Science of Food and Agriculture 32:493-497.

Frazier, W.C. and Westhoff, D.C. (1978). Food microbiology. $3^{\text {rd }}$ ed., McGraw-Hill Book Company, U.S.A. p. 30-31. 
Ghazali, H.M. (1990). Production and characterization of cell-bound lipases secreted by a newly isolated strain of Geotrichum candidum. PhD. Thesis. University Putra Malaysia.

Gunstone, F.D. (2004). Basic oleochemicals, oleochemical products and new industrial oils. In: Oleochemical Manufacture and Applications. F.D. Gunstone and R.J. Hamilton. (eds). Sheffield Academic Press, Sheffield, p. 1-19.

Hiol, A., Jonzo, M.D., Druet, D., and Comeau, L.C. (1999). Production, purification and characterization of an extracellular lipase from Mucor hiemalis $f$. hiemalis. Enzyme and Microbial Technology. 25:8087.

Iwai, M., Tsujisaka, Y., Okamoto Y. and Fukumoto J. (1973). Lipid requirement for the lipase production of Geotrichum candidum Link. Agric. Biol. Chem. 37(4):929-931.

Jacobsen, T., Olsen, J. and Allermann, K. (1989). Production, partial purification, and immunochemical characterization of multiple forms of lipase from Geotrichum candidum. Enzyme and Microbial Technology 11:90-95.

Kreger-van Rij, N. J. W. (1984). The Genera Endomyces and Geotrichum. In: Kreger-van Rij, N. J. W. (ed.) The Yeast: A Taxanomic Study, $3^{\text {rd }}$ ed. Elsevier Science, Amsterdam, p.13-15.

Lodder, J. (1971).The Yeasts: A Taxonomic Study. NorthHolland Publishing Co., Amsterdam, p. 34-120, 13071347.

Pantzaris T.P. (1995). Pocketbook of palm oil uses. Palm Oil Research Institute of Malaysia (PORIM), Ministry of Primary Industry, p.122-151.

Shimada, Y., Maruyama, K., Nakamura, M., Nakayama, S., Sughihara, A. and Tominaga, Y. (1995). Selective hydrolysis of polyunsaturated fatty acidcontaining oil with Geotrichum candidum lipase. J. Am. Oil Chem. Soc. 72(12):1577-1581.
Shridhar R., Lakshminarayana G. and Kaimal T.N.B. (1991). Modification of selected vegetable oils to high oleic oils by lipase-catalyzed ester interchange. Journal of Agricultural Food Chemistry. 39:20692071.

Sidebottom, C.M., Charton, E., Dunn, P.P.J., Mycock, G., Davies, C., L. Sutton, Macrae, A.R. and Slabas, A.R. (1991). Geotrichum candidum produces several lipases with markedly different substrate specificity. European Journal of Biochemistry. 202:485-491.

Siew, W.L., Tang, T.S. and Tan, Y. A. (1995). Methods of test for palm oil and palm oil products. Palm Oil Research Institute of Malaysia, Ministry of Primary Industries, Malaysia, p.40-42.

Sonnet P.E., Foglia T.A. and Baillargeon. (1993). Fatty acid selectivity: The selectivity of lipases of Geotrichum candidum. J. Am. Oil Chem. Soc. 70(10):1043-1045

Sonntag, N.O.V. (1991) Erucic, behenic: Feedstocks of the $21^{\text {st }}$ century. INFORM 2(5):449-463.

Sugiura, M. and M. Isobe (1975). Studies on the lipase of Chromobacterium viscosum . IV. Substrate specificity of a low molecular weight lipase. Chemical and Pharmaceutical Bulletin. 23(6):1226-1230.

Tan, K.H. and Gill, C.O. (1984). Effect of culture conditions on batch growth of Saccharomycopsis lipolytica on olive oil. Applied Microbiology and Biotechnology. 20:201-206.

Tsujisaka, Y., Iwai, M., Fukumota, J. and Okamato, Y. (1973). Induced formation of lipase by Geotrichum candidum Link. Agric. Biol. Chem. 37(4):837-842.

van der Walt, J.P. and Yarrow, D. (1984). Methods for the isolation, maintenance, classification and identification of yeasts. In: Kreger-van Rij, N.J.W. (ed.) The Yeast: A Taxonomic Study. Elsevier Science, Amsterdam, p. 45-104. 